\title{
Correlation Function of Fields in One-Dimensional Bose-Gas
}

\author{
V. E. Korepin and N. A. Slavnov \\ Institute for Theoretical Physics, State University of New York at Stony Brook, Stony Brook, \\ NY 11794-3840, USA
}

Received September 10, 1990

\begin{abstract}
Correlation function of fields is presented as a Fredholm minor, at finite coupling constant in one-dimensional Bose gas.
\end{abstract}

\section{Introduction}

We discuss correlation function of fields in the quantum nonlinear Schrödinger equation model (NS-model). The Hamiltonian of this model is equal to

$$
\mathscr{H}=\int_{0}^{L} d x\left(\partial_{x} \psi^{+} \partial_{x} \psi+c \psi^{+} \psi^{+} \psi \psi-h \psi^{+} \psi\right)
$$

Here $c>0$ is a coupling constant, $h>0$ : chemical potential; $L$ : a length of a box; $\psi(x)$ : a canonical Bose-field:

$$
\begin{aligned}
{\left[\psi(x), \psi^{+}(y)\right] } & =\delta(x-y), \\
\psi(x)|0\rangle & =0
\end{aligned}
$$

In the limit $c=\infty$ (free fermions) the correlator was calculated by Lenard [1] in terms of a Fredholm minor. This representation was used for writing differential equations for the correlator $[2,3]$. In the present paper we consider the case of the finite coupling constant $c$. Using the method of algebraic anzats Bethe we present the correlator as a minor of an integral operator, which depends on auxiliary quantum fields. Such a representation can be used for writing the system of integro-differential equations for the correlation function.

\section{Algebraic Anzats Bethe}

The main object of algebraic anzats Bethe is the monodromy matrix $T(\lambda)$. In the case of NS-model it is $2 \times 2$ matrix:

$$
T(\lambda)=\left(\begin{array}{ll}
A(\lambda) & B(\lambda) \\
C(\lambda) & D(\lambda)
\end{array}\right)
$$


Matrix elements are quantum operators, which depend on the spectral parameter $\lambda$. Commutation relations between these operators are given by the formula:

$$
R(\lambda, \mu)(T(\lambda) \otimes T(\mu))=(T(\mu) \otimes T(\lambda)) R(\lambda, \mu) .
$$

Here $R(\lambda, \mu)$ is a $4 \times 4$ matrix with $c$-number elements

$$
R(\lambda, \mu)=\left(\begin{array}{cccc}
f(\mu, \lambda) & 0 & 0 & 0 \\
0 & g(\mu, \lambda) & 1 & 0 \\
0 & 1 & g(\mu, \lambda) & 0 \\
0 & 0 & 0 & f(\mu, \lambda)
\end{array}\right)
$$

where

$$
f(\lambda, \mu)=\frac{\lambda-\mu+i c}{\lambda-\mu} ; \quad g(\lambda, \mu)=\frac{i c}{\lambda-\mu}
$$

The functions

$$
\begin{aligned}
& h(\lambda, \mu)=\frac{f(\lambda, \mu)}{g(\lambda, \mu)}=\frac{\lambda-\mu+i c}{i c}, \\
& t(\lambda, \mu)=\frac{g(\lambda, \mu)}{h(\lambda, \mu)}=-\frac{c^{2}}{(\lambda-\mu)(\lambda-\mu+i c)}
\end{aligned}
$$

will also be useful.

Let us write down some of the commutation relations (2.2) explicitly:

$$
\begin{aligned}
{[B(\lambda), B(\mu)] } & =[C(\lambda), C(\mu)]=0, \\
A(\mu) B(\lambda) & =f(\mu, \lambda) B(\lambda) A(\mu)+g(\lambda, \mu) B(\mu) A(\lambda), \\
C(\mu) D(\lambda) & =f(\mu, \lambda) D(\lambda) C(\mu)+g(\lambda, \mu) D(\mu) C(\lambda), \\
{[C(\mu), B(\lambda)] } & =g(\mu, \lambda)\{A(\mu) D(\lambda)-A(\lambda) D(\mu)\} .
\end{aligned}
$$

Other important objects in algebraic anzats Bethe are the pseudovacuum $|0\rangle$ and dual pseudovacuum $\langle 0|$. In the NS-model these vectors coincide with vectors (1.2), so we use the same notations for them. Properties of $|0\rangle$ and $\langle 0|$ are the following:

$$
\begin{aligned}
& A(\lambda)|0\rangle=a(\lambda)|0\rangle ; \quad D(\lambda)|0\rangle=d(\lambda)|0\rangle ; \quad C(\lambda)|0\rangle=0, \\
& \langle 0| A(\lambda)=a(\lambda)\langle 0| ; \quad\langle 0| D(\lambda)=d(\lambda)\langle 0| ; \quad\langle 0| B(\lambda)=0 .
\end{aligned}
$$

Here $a(\lambda)=\exp \left\{-\frac{i \lambda L}{2}\right\}, d(\lambda)=a^{-1}(\lambda)$. Eigenfunctions of Hamiltonian (1.1) coincide with eigenfunctions of transfer-matrix $\tau(\lambda)=A(\lambda)+D(\lambda)$. They can be written in the form

$$
\left|\Psi_{N}(\{\lambda\})\right\rangle=\prod_{j=1}^{N} B\left(\lambda_{j}\right)|0\rangle,
$$

where all parameters $\lambda_{j}$ are different and satisfy the system

$$
\frac{a\left(\lambda_{j}\right)}{d\left(\lambda_{j}\right)} \prod_{k=1}^{N} \frac{h\left(\lambda_{j}, \lambda_{k}\right)}{h\left(\lambda_{k}, \lambda_{j}\right)}=(-1)^{N-1} .
$$

The function

$$
\left\langle\tilde{\Psi}_{N}(\{\lambda\})\right|=\langle 0| \prod_{j=1}^{N} C\left(\lambda_{j}\right),
$$


where $\lambda_{j}$ also satisfy (2.13), is a dual eigenfunction.

To describe commutation relations between fields $\psi^{+}(x), \psi(x)$ and the operators $A, B, C, D$ we use a lattice approximation of the model. The monodromy matrix (2.1) is given by a product of $L$-operators

$$
T(\lambda)=L_{M}(\lambda) L_{M-1}(\lambda) \ldots L_{1}(\lambda),
$$

where

$$
L_{n}(\lambda)=\left(\begin{array}{cc}
1-\frac{i \lambda \Delta}{2} & -i \sqrt{c} \Delta \psi_{n}^{+} \\
i \sqrt{c} \Delta \psi_{n} & 1+\frac{i \lambda \Delta}{2}
\end{array}\right)+O\left(\Delta^{2}\right) .
$$

$\Delta$ is a step of the lattice. Operators $\psi_{n}^{+}, \psi_{n}$ are lattice approximations of the fields $\psi^{+}(x)$ and $\psi(x)$.

Their commutator is equal to

$$
\left[\psi_{n}, \psi_{m}^{+}\right]=\frac{1}{\Delta} \delta_{n m} .
$$

Let us represent $T(\lambda)$ by the following way:

$$
T(\lambda)=T_{2}(\lambda) T_{1}(\lambda) .
$$

Here

$$
T_{2}(\lambda)=L_{M}(\lambda) \ldots L_{n}(\lambda) ; \quad T_{1}(\lambda)=L_{n-1}(\lambda) \ldots L_{1}(\lambda) .
$$

$n$ is a fixed site of the lattice and

$$
T_{i}(\lambda)=\left(\begin{array}{ll}
A_{i}(\lambda) & B_{i}(\lambda) \\
C_{i}(\lambda) & D_{i}(\lambda)
\end{array}\right), \quad i=1,2 .
$$

Using (2.16) one can obtain

$$
\left[T_{2}(\lambda), \psi_{n}^{+}\right]=i \sqrt{c} L_{M}(\lambda) \ldots L_{n+1}(\lambda)\left(\begin{array}{ll}
0 & 0 \\
1 & 0
\end{array}\right) .
$$

In the continuous limit $\Delta \rightarrow 0, M \rightarrow \infty, M \Delta=L$ we can rewrite this formula

$$
\left[T_{2}(\lambda), \psi^{+}(x)\right]=i \sqrt{c} T_{2}(\lambda)\left(\begin{array}{cc}
0 & 0 \\
1 & 0
\end{array}\right) .
$$

Here the point $x$ corresponds to the $n^{\text {th }}$ site of lattice. Finally we have

$$
\left[C_{2}(\lambda), \psi^{+}(x)\right]=i \sqrt{c} D_{2}(\lambda) .
$$

Commutators $\psi^{+}(x)$ with elements of the matrix $T_{1}(\lambda)$ are equal to zero. Commutator between $\psi(0)$ and $T(\lambda)$ can be calculated in a similar way. Namely

$$
[\psi(0), B(\lambda)]=-i \sqrt{c} A(\lambda) .
$$

The properties (2.22), (2.23) are sufficient for calculation of the correlation function of the fields. At the conclusion of the section we'll give some properties of the matrices $T_{1}(\lambda)$ and $T_{2}(\lambda)$. Each of them satisfies Eq. (2.2) with the $R$-matrix (2.3), so commutation relations (2.7)-(2.10) for operators $A_{i}, B_{i}, C_{i}, D_{i}(i=1,2)$ are valid. The elements of the different matrix commute. Matrix $T_{i}(\lambda)$ has a pseudovacuum $|0\rangle_{i}$ and dual vector ${ }_{i}\langle 0|$. The pseudovacuum $|0\rangle$ is equal to 
$|0\rangle=|0\rangle_{2} \otimes|0\rangle_{1}$. The properties of vectors $|0\rangle_{i}$ and ${ }_{i}\langle 0|$ are similar to properties of $|0\rangle$ and $\langle 0|$ :

$$
\begin{array}{lll}
A_{i}(\lambda)|0\rangle_{i}=a_{i}(\lambda)|0\rangle_{i} ; & D_{i}(\lambda)|0\rangle_{i}=d_{i}(\lambda)|0\rangle_{i} ; & C_{i}(\lambda)|0\rangle_{i}=0, \\
{ }_{i}\langle 0| A_{i}(\lambda)=a_{i}(\lambda)_{i}\langle 0| ; & { }_{i}\langle 0| D_{i}(\lambda)=d_{i}(\lambda)_{i}\langle 0| ; & { }_{i}\langle 0| B_{i}(\lambda)=0 .
\end{array}
$$

Here $a_{1}(\lambda)=\exp \left\{-\frac{i x \lambda}{2}\right\}, a_{2}(\lambda)=\exp \left\{\frac{i \lambda}{2}(L-x)\right\} ; d_{i}(\lambda)=a_{i}^{-1}(\lambda)$.

Note that

$$
a(\lambda)=a_{1}(\lambda) a_{2}(\lambda), \quad d(\lambda)=d_{1}(\lambda) d_{2}(\lambda)
$$

Finally we'll give a representation for the function $\prod_{j=1}^{N} B\left(\lambda_{j}\right)|0\rangle$ in terms of the
ements of matrices $T_{1}$ and $T_{2}$ (see [4]):

$$
\begin{aligned}
\prod_{j=1}^{N} B\left(\lambda_{j}\right)|0\rangle= & \sum_{\{\lambda\}=\left\{\lambda_{\mathrm{I}}\right\} \cup\left\{\lambda_{\mathrm{II}}\right\}} \prod_{\mathrm{I}} B_{1}\left(\lambda_{\mathrm{I}}\right)|0\rangle_{1} \prod_{\mathrm{II}} B_{2}\left(\lambda_{\mathrm{II}}\right)|0\rangle_{2} \\
& \times \prod_{\mathrm{I}} a_{2}\left(\lambda_{\mathrm{I}}\right) \prod_{\mathrm{II}} d_{1}\left(\lambda_{\mathrm{II}}\right) \prod_{\mathrm{I}, \mathrm{II}} f\left(\lambda_{\mathrm{I}}, \lambda_{\mathrm{II}}\right) .
\end{aligned}
$$

Here the sum is taken over all partitions of the set $\{\lambda\}$ into two disjoint subsets $\left\{\lambda_{\mathrm{I}}\right\}$ and $\left\{\lambda_{\mathrm{II}}\right\}$. The symbol $\prod_{\mathrm{I}}\left(\right.$ or $\left.\prod_{\mathrm{II}}\right)$ means the product over all
$\lambda \in\left\{\lambda_{\mathrm{I}}\right\}$ (correspondingly $\left.\lambda \in\left\{\lambda_{\mathrm{II}}\right\}\right)$.

An analogous formula can be written for the dual function:

$$
\begin{aligned}
\langle 0| \prod_{j=1}^{N} C\left(\lambda_{j}\right)= & \sum_{\{\lambda\}=\left\{\lambda_{\mathrm{I}}\right\} \cup\left\{\lambda_{\mathrm{II}}\right\}}{ }_{1}\langle 0| \prod_{\mathrm{I}} C_{1}\left(\lambda_{\mathrm{I}}\right)_{2}\langle 0| \prod_{\mathrm{II}} C_{2}\left(\lambda_{\mathrm{II}}\right) \\
& \times \prod_{\mathrm{I}} d_{2}\left(\lambda_{\mathrm{I}}\right) \prod_{\mathrm{II}} a_{1}\left(\lambda_{\mathrm{II}}\right) \prod_{\mathrm{I}, \mathrm{II}} f\left(\lambda_{\mathrm{II}}, \lambda_{\mathrm{I}}\right) .
\end{aligned}
$$

\section{Matrix Element of Operators $\psi^{+}(x) \psi(0)$}

In this section we'll calculate the matrix element of the operator $\psi^{+}(x) \psi(0)$ :

$$
G_{N}=\left\langle 0\left|\prod_{j=1}^{N} C\left(\lambda_{j}^{C}\right) \psi^{+}(x) \psi(0) \prod_{j=1}^{N} B\left(\lambda_{j}^{B}\right)\right| 0\right\rangle .
$$

Here the parameters $\left\{\lambda^{C}\right\}$ and $\left\{\lambda^{B}\right\}$ are arbitrary complex numbers. The only condition is $\lambda_{j}^{B} \neq \lambda_{K}^{B}, \lambda_{j}^{C} \neq \lambda_{K}^{C},(j, K=1, \ldots, N)$. Consider the action of the operator $\psi(0)$ on the vector $\prod_{j=1}^{N} B\left(\lambda_{j}\right)|0\rangle$. Using (2.23) we have

$$
\psi(0) \prod_{j=1}^{N} B\left(\lambda_{j}\right)|0\rangle=-i \sqrt{c} \sum_{K=1}^{N} B\left(\lambda_{1}\right) \ldots B\left(\lambda_{K-1}\right) A\left(\lambda_{K}\right) B\left(\lambda_{K+1}\right) \ldots B\left(\lambda_{N}\right)|0\rangle .
$$


One can rewrite this formula as follows [see (2.8), (2.11)]:

$$
\psi(0) \prod_{i=1}^{N} B\left(\lambda_{j}\right)|0\rangle=-i \sqrt{c} \sum_{K=1}^{N} \Lambda_{K} a\left(\lambda_{K}\right) \prod_{\substack{m=1 \\ m \neq K}}^{N} B\left(\lambda_{m}\right)|0\rangle
$$

where $\Lambda_{K}$ is a rational function on $\lambda$ 's, depending on the functions $f$ and $g$ (2.4). Let us calculate this coefficient. Due to (2.7) the right-hand side of (3.3) is symmetric in all $\lambda$, so it is sufficient to calculate the coefficient $\Lambda_{1}$. Obviously this term can be obtained only if $K=1$ in (3.2):

$$
-i \sqrt{c} A\left(\lambda_{1}\right) B\left(\lambda_{2}\right) \ldots B\left(\lambda_{N}\right) \text {. }
$$

Now we must move the operator $A\left(\lambda_{1}\right)$ to the right, using only the first term in formula (2.8). We have

$$
\Lambda_{1}=\prod_{m=2}^{N} f\left(\lambda_{1}, \lambda_{m}\right)
$$

and so

$$
\Lambda_{K}=\prod_{\substack{m=1 \\ m \neq K}}^{N} f\left(\lambda_{K}, \lambda_{m}\right)
$$

Consider now the action $\psi^{+}(x)$ on the vector $\langle 0| \prod_{j=1}^{N} C\left(\lambda_{j}\right)$. To do it, it is necessary to represent this vector in terms of $C_{1}$ and $C_{2}$ (2.27). After that all calculations are analogous to the case already considered. Formula (2.22) shows that

$$
\begin{aligned}
\langle 0| \prod_{j=1}^{N} C\left(\lambda_{j}\right) \psi^{+}(x)= & i \sqrt{c} \sum_{\{\lambda\}=\left\{\lambda_{0}\right\} \cup\left\{\lambda_{\mathrm{I}}\right\} \cup\left\{\lambda_{\mathrm{II}}\right\}} a_{1}\left(\lambda_{0}\right) d_{2}\left(\lambda_{0}\right) \\
& \times{ }_{1}\langle 0| \prod_{\mathrm{I}} C_{1}\left(\lambda_{\mathrm{I}}\right)_{2}\langle 0| \prod_{\mathrm{II}} C_{2}\left(\lambda_{\mathrm{II}}\right) \prod_{\mathrm{I}} d_{2}\left(\lambda_{\mathrm{I}}\right) f\left(\lambda_{0}, \lambda_{\mathrm{I}}\right) \\
& \times \prod_{\mathrm{II}} a_{1}\left(\lambda_{\mathrm{II}}\right) f\left(\lambda_{\mathrm{II}}, \lambda_{0}\right) \prod_{\mathrm{I}, \mathrm{II}} f\left(\lambda_{\mathrm{II}}, \lambda_{\mathrm{I}}\right) .
\end{aligned}
$$

Here the sum is taken over three subsets $\left\{\lambda_{0}\right\},\left\{\lambda_{\mathrm{I}}\right\},\left\{\lambda_{\mathrm{II}}\right\}$. Subsets $\left\{\lambda_{\mathrm{I}}\right\},\left\{\lambda_{\mathrm{II}}\right\}$ are arbitrary, subset $\left\{\lambda_{0}\right\}$ contains exactly one element.

Formula (3.3) also can be written in terms of $B_{1}$ and $B_{2}$. Combining this formula with (3.5) we find matrix element $G_{N}$ :

$$
\begin{aligned}
c^{-1} G_{N}= & \sum_{\substack{\left\{\lambda^{C}\right\}=\left\{\lambda_{0}^{C}\right\} \cup\left\{\lambda_{\mathrm{I}}^{C}\right\} \cup\left\{\lambda_{\mathrm{II}}^{C}\right\} \\
\left\{\lambda^{B}\right\}=\left\{\lambda_{0}^{B}\right\} \cup\left\{\lambda_{\mathrm{I}}^{B}\right\} \cup\left\{\lambda_{\mathrm{II}}^{B}\right\}}}\left\langle 0\left|\prod_{\mathrm{I}} C_{1}\left(\lambda_{\mathrm{I}}^{C}\right) \prod_{\mathrm{I}} B_{1}\left(\lambda_{\mathrm{I}}^{B}\right)\right| 0\right\rangle_{1} \\
& \times{ }_{2}\left\langle 0\left|\prod_{\mathrm{II}} C_{2}\left(\lambda_{\mathrm{II}}^{C}\right) \prod_{\mathrm{II}} B_{2}\left(\lambda_{\mathrm{II}}^{B}\right)\right| 0\right\rangle_{2} a_{1}\left(\lambda_{0}^{C}\right) d_{2}\left(\lambda_{0}^{C}\right) a_{1}\left(\lambda_{0}^{B}\right) a_{2}\left(\lambda_{0}^{B}\right) \\
& \times \prod_{\mathrm{I}}\left\{a_{2}\left(\lambda_{\mathrm{I}}^{B}\right) d_{2}\left(\lambda_{\mathrm{I}}^{C}\right) f_{0 \mathrm{I}}^{C C} f_{0 \mathrm{I}}^{B B}\right\} \prod_{\mathrm{II}}\left\{d_{1}\left(\lambda_{\mathrm{II}}^{B}\right) a_{1}\left(\lambda_{\mathrm{II}}^{C}\right) f_{\mathrm{II} 0}^{C C} f_{0 \mathrm{II}}^{B B}\right\} \\
& \times \prod_{\mathrm{I}, \mathrm{II}}\left\{f_{\mathrm{I}, \mathrm{II}}^{B B} f_{\mathrm{II}, \mathrm{I}}^{C C}\right\} .
\end{aligned}
$$


Here partitions of the sets $\left\{\lambda^{C}\right\}$ and $\left\{\lambda^{B}\right\}$ are independent except that $\operatorname{card}\left\{\lambda_{I}^{C}\right\}=\operatorname{card}\left\{\lambda_{I}^{B}\right\}, \operatorname{card}\left\{\lambda_{0}^{C}\right\}=\operatorname{card}\left\{\lambda_{0}^{B}\right\}=1$. In (3.6) we also use abbreviated notations:

$$
f_{\mathrm{I}, \mathrm{II}}^{B B}=f\left(\lambda_{\mathrm{I}}^{B}, \lambda_{\mathrm{II}}^{B}\right), \quad f_{0 \mathrm{I}}^{C C}=f\left(\lambda_{0}^{C}, \lambda_{\mathrm{I}}^{C}\right)
$$

etc. Such notations as

$$
h_{\mathrm{I}, \mathrm{II}}^{C B}=h\left(\lambda_{\mathrm{I}}^{C}, \lambda_{\mathrm{II}}^{B}\right), \quad g_{j K}^{C C}=g\left(\lambda_{j}^{C}, \lambda_{K}^{C}\right), \quad t_{j K}=t\left(\lambda_{j}, \lambda_{K}\right)
$$

and others we'll use in the next section.

So we expressed the matrix element $G_{N}$ in terms of scalar products

$$
\left\langle 0\left|\prod C\left(\lambda^{C}\right) \prod B\left(\lambda^{B}\right)\right| 0\right\rangle
$$

\section{Dual Fields}

To write formula (3.6) as a determinant of $N \times N$ matrix we'll use the technique of dual fields. This approach was developed in [5]. Let us introduce 10 new fields. Each of them is the sum of operator "coordinate" and operator "momentum":

$$
\begin{aligned}
\Phi_{A_{K}}(\lambda) & =Q_{A_{K}}(\lambda)+P_{D_{K}}(\lambda) ; & \Phi_{D_{K}}(\lambda) & =Q_{D_{K}}(\lambda)+P_{A_{K}}(\lambda) ; \quad K=1,2, \\
\varphi_{A_{1}}(\lambda) & =q_{A_{1}}(\lambda)+p_{D_{2}}(\lambda) ; & & \varphi_{D_{1}}(\lambda)=q_{D_{1}}(\lambda)+p_{A_{2}}(\lambda), \\
\varphi_{A_{2}}(\lambda) & =q_{A_{2}}(\lambda)+p_{D_{1}}(\lambda) ; & & \varphi_{D_{2}}(\lambda)=q_{D_{2}}(\lambda)+p_{A_{1}}(\lambda) \\
\varphi_{A_{3}}(\lambda) & =q_{A_{3}}(\lambda)+p_{D_{3}}(\lambda) ; & & \varphi_{D_{3}}(\lambda)=q_{D_{3}}(\lambda)+p_{A_{3}}(\lambda) .
\end{aligned}
$$

These are Bose fields.

The fields $\Phi$ and $\varphi$ act in auxiliary Fock space. Vacuum in this space also will be denoted by $|0\rangle$. All "momenta" annihilate it:

$$
P(\lambda)|0\rangle=p(\lambda)|0\rangle=0 .
$$

The dual vacuum $\langle 0|$ is the eigenvector for "coordinates":

$$
\begin{aligned}
\langle 0| Q_{A_{K}}(\lambda) & =\langle 0| q_{A_{K}}(\lambda)=\ln a_{K}(\lambda)\langle 0|, \quad K=1,2, \\
\langle 0| Q_{D_{K}}(\lambda) & =\langle 0| q_{D_{K}}(\lambda)=\ln d_{K}(\lambda)\langle 0|, \quad K=1,2, \\
\langle 0| q_{A_{3}}(\lambda) & =\langle 0| q_{D_{3}}(\lambda)=0, \\
\langle 0 \mid 0\rangle & =1 .
\end{aligned}
$$

Nonzero commutators are

$$
\begin{aligned}
& \begin{array}{l}
{\left[P_{A_{j}}(\lambda), Q_{A_{K}}(\mu)\right]=\delta_{j K} \ln h(\mu, \lambda)} \\
{\left[P_{D_{j}}(\lambda), Q_{D_{K}}(\mu)\right]=\delta_{j K} \ln h(\lambda, \mu)}
\end{array} ; \quad j, K=1,2, \\
& \begin{array}{l}
{\left[p_{A_{j}}(\lambda), q_{A_{K}}(\mu)\right]=\delta_{j K} \ln h(\mu, \lambda)} \\
{\left[p_{D_{j}}(\lambda), q_{D_{K}}(\mu)\right]=\delta_{j K} \ln h(\lambda, \mu)}
\end{array} ; \quad j, K=1,2,3 .
\end{aligned}
$$

The remarkable property of the fields $\Phi, \varphi$ is that they all commute:

$$
\left[\Phi_{\alpha}(\lambda), \Phi_{\beta}(\lambda)\right]=\left[\Phi_{\alpha}(\lambda), \varphi_{\beta}(\mu)\right]=\left[\varphi_{\alpha}(\lambda), \varphi_{\beta}(\mu)\right]=0 .
$$

Here $\alpha, \beta$ run throw all the possible indices. 
One of the results of paper [5] is the representation of scalar product in terms of dual fields $\Phi$ :

$$
\begin{aligned}
{ }_{m}\langle 0| & \prod_{j=1}^{N} C_{m}\left(\lambda_{j}^{C}\right) \prod_{j=1}^{N} B_{m}\left(\lambda_{j}^{B}\right)|0\rangle_{m} \\
& =\prod_{j>K}^{N} g_{j K}^{C C} g_{K j}^{B B}\left\langle 0\left|\operatorname{det}_{N} S^{(m)}\left(\lambda^{C}, \lambda^{B}\right)\right| 0\right\rangle, \quad m=1,2 .
\end{aligned}
$$

Here $S^{(m)}\left(\lambda^{C}, \lambda^{B}\right)$ is an $N \times N$ matrix with elements

$$
\begin{aligned}
S_{j K}^{(m)}\left(\lambda^{C}, \lambda^{B}\right)= & t_{j K}^{C B} \exp \left\{\Phi_{A_{m}}\left(\lambda_{j}^{C}\right)+\Phi_{D_{m}}\left(\lambda_{K}^{B}\right)\right\} \\
& +t_{K j}^{B C} \exp \left\{\Phi_{D_{m}}\left(\lambda_{j}^{C}\right)+\Phi_{A_{m}}\left(\lambda_{K}^{B}\right)\right\} .
\end{aligned}
$$

Recall that the notations $g_{j K}^{C C}, t_{j K}^{C B}$ mean $g\left(\lambda_{j}^{C}, \lambda_{K}^{C}\right), t\left(\lambda_{j}^{C}, \lambda_{K}^{B}\right)$ correspondingly. Using (4.9) one can write (3.6) in such a way

$$
\begin{aligned}
c^{-1} G_{N}= & \prod_{j>K}^{N} g_{j K}^{C C} g_{K j}^{B B} \sum_{\substack{\left\{\lambda^{C}\right\}=\left\{\lambda_{0}^{C}\right\} \cup\left\{\lambda_{\mathrm{I}}^{C}\right\} \cup\left\{\lambda_{\mathrm{II}}^{C}\right\} \\
\left\{\lambda^{B}\right\}=\left\{\lambda_{0}^{B}\right\} \cup\left\{\lambda_{\mathrm{I}}^{B}\right\} \cup\left\{\lambda_{\mathrm{II}}^{B}\right\}}}(-1)^{\left[P_{C}+P_{B}\right]+N_{1}} \\
& \times\left\langle 0\left|\operatorname{det}_{N_{1}} S^{(1)}\left(\lambda_{\mathrm{I}}^{C}, \lambda_{\mathrm{I}}^{B}\right) \operatorname{det}_{N_{2}} S^{(2)}\left(\lambda_{\mathrm{II}}^{C}, \lambda_{\mathrm{II}}^{B}\right)\right| 0\right\rangle \\
& \times a_{1}\left(\lambda_{0}^{C}\right) d_{2}\left(\lambda_{0}^{C}\right) a_{1}\left(\lambda_{0}^{B}\right) a_{2}\left(\lambda_{0}^{B}\right) \prod_{I}\left\{a_{2}\left(\lambda_{\mathrm{I}}^{B}\right) d_{2}\left(\lambda_{\mathrm{I}}^{C}\right) h_{0 \mathrm{I}}^{C C} h_{0 \mathrm{I}}^{B B}\right\} \\
& \times \prod_{\mathrm{II}}\left\{d_{1}\left(\lambda_{\mathrm{II}}^{B}\right) a_{1}\left(\lambda_{\mathrm{II}}^{C}\right) h_{\mathrm{II} 0}^{C C} h_{0 \mathrm{II}}^{B B}\right\} \prod_{\mathrm{I}, \mathrm{II}}\left\{h_{\mathrm{II}, \mathrm{I}}^{C C} h_{\mathrm{I}, \mathrm{II}}^{B B}\right\} .
\end{aligned}
$$

Here we write all functions $f(\lambda, \mu)$ as a product $g(\lambda, \mu) h(\lambda, \mu)$ [see (2.5)] and use property of antisymmetry of functions $g(\lambda, \mu) . P_{C, B}$ is permutation, which transforms sequence $\left\{\lambda_{0}^{C, B}\right\},\left\{\lambda_{\mathrm{I}}^{C, B}\right\},\left\{\lambda_{\mathrm{II}}^{C, B}\right\}$ into sequence $\left\{\lambda_{1}^{C, B}, \ldots, \lambda_{N}^{C, B}\right\}$,

$$
N_{1}=\operatorname{card}\left\{\lambda_{\mathrm{I}}^{C}\right\}, \quad N_{2}=\operatorname{card}\left\{\lambda_{\mathrm{II}}^{C}\right\}=N-N_{1}-1 \text {. }
$$

Now everything is ready to prove the following theorem.

Theorem. The matrix element $G_{N}$ of operator $\psi^{+}(x) \psi(0)$ is equal to

$$
G_{N}=\left.\prod_{j>K} g_{j K}^{C C} g_{K j}^{B B} \frac{\partial}{\partial \alpha}\left\langle 0\left|\operatorname{det}_{N} M\right| 0\right\rangle\right|_{\alpha=0}
$$

Here $M$ is an $N \times N$ matrix:

$$
\begin{aligned}
M_{j K}= & S_{j K}^{(2)}\left(\lambda_{j}^{C}, \lambda_{K}^{B}\right) \exp \left\{\varphi_{A_{1}}\left(\lambda_{j}^{C}\right)+\varphi_{D_{1}}\left(\lambda_{K}^{B}\right)\right\} \\
& -S_{j K}^{(1)}\left(\lambda_{j}^{C}, \lambda_{K}^{B}\right) \exp \left\{\varphi_{D_{2}}\left(\lambda_{j}^{C}\right)+\varphi_{A_{2}}\left(\lambda_{K}^{B}\right)+\varphi_{D_{3}}\left(\lambda_{K}^{B}\right)\right\} \\
& +c \alpha a_{1}\left(\lambda_{K}^{B}\right) \exp \left\{\varphi_{A_{3}}\left(\lambda_{K}^{B}\right)+\varphi_{A_{2}}\left(\lambda_{K}^{B}\right)\right. \\
& \left.+\varphi_{A_{1}}\left(\lambda_{j}^{C}\right)+\varphi_{D_{2}}\left(\lambda_{j}^{C}\right)\right\} .
\end{aligned}
$$


Proof. One should write $\operatorname{det}_{N} M$ as a determinant of the sum of three matrices. Calculating the derivative of $\alpha$ we have

$$
\begin{aligned}
\frac{\partial}{\partial \alpha}\left\langle 0\left|\operatorname{det}_{N} M\right| 0\right\rangle= & \sum_{\substack{\left\{\lambda^{C}\right\}=\left\{\lambda_{0}^{C}\right\} \cup\left\{\lambda_{I}^{C}\right\} \cup\left\{\lambda_{\mathrm{II}}^{C}\right\} \\
\left\{\lambda^{B}\right\}=\left\{\lambda_{0}^{B}\right\} \cup\left\{\lambda_{1}^{B}\right\} \cup\left\{\lambda_{\mathrm{II}}^{B}\right\}}}(-1)^{\left[P_{C}+P_{B}\right]+N_{1}} \\
& \times\langle 0| \operatorname{det}_{N_{1}} S^{(1)}\left(\lambda_{\mathrm{I}}^{C}, \lambda_{\mathrm{I}}^{B}\right) \operatorname{det}_{N_{2}} S^{(2)}\left(\lambda_{\mathrm{II}}^{C}, \lambda_{\mathrm{II}}^{B}\right) \cdot c a_{1}\left(\lambda_{0}^{B}\right) \\
& \times \exp \left\{\varphi_{A_{3}}\left(\lambda_{0}^{B}\right)+\varphi_{A_{2}}\left(\lambda_{0}^{B}\right)+\varphi_{A_{1}}\left(\lambda_{0}^{C}\right)+\varphi_{D_{2}}\left(\lambda_{0}^{C}\right)\right\} \\
& \times \prod_{\mathrm{II}} \exp \left\{\varphi_{A_{1}}\left(\lambda_{\mathrm{II}}^{C}\right)+\varphi_{D_{1}}\left(\lambda_{\mathrm{II}}^{B}\right)\right\} \prod_{\mathrm{II}} \exp \left\{\varphi_{D_{2}}\left(\lambda_{\mathrm{II}}^{C}\right)\right. \\
& \left.+\varphi_{A_{2}}\left(\lambda_{\mathrm{II}}^{B}\right)+\varphi_{D_{3}}\left(\lambda_{\mathrm{II}}^{B}\right)\right\}|0\rangle .
\end{aligned}
$$

Calculating the vacuum mean value of the products $e^{\varphi}$ we obtain formula (4.11), which completes the proof.

So the matrix element $\psi^{+}(x) \psi(0)$ is represented as a determinant of the $N \times N$ matrix.

\section{Correlation Function of Fields}

Now let us use (4.12) to calculate the correlator in the $N$-particle state. To do it one should put $\lambda_{j}^{C}=\lambda_{j}^{B}=\lambda_{j}$ in (4.12), (4.13) and demand the $\{\lambda\}$ satisfy system (2.13). First of all we transform the determinant:

$$
\begin{aligned}
& \left\langle 0\left|\operatorname{det}_{N} M\right| 0\right\rangle \\
& =\langle 0| \prod_{m=1}^{N} \exp \left\{\Phi_{A_{2}}\left(\lambda_{m}^{C}\right)+\Phi_{D_{2}}\left(\lambda_{m}^{B}\right)+\varphi_{A_{1}}\left(\lambda_{m}^{C}\right)+\varphi_{D_{1}}\left(\lambda_{m}^{B}\right) \operatorname{det}_{N} \tilde{M}|0\rangle\right. \\
& =\prod_{m=1}^{N} a\left(\lambda_{m}^{C}\right) d\left(\lambda_{m}^{B}\right) \prod_{m, e=1}^{N} h_{m e}^{C B}\left\langle\tilde{0}\left|\operatorname{det}_{N} \tilde{M}\right| 0\right\rangle,
\end{aligned}
$$

where

$$
\begin{gathered}
\langle\tilde{0}|=\langle 0| \prod_{m=1}^{N} \exp \left\{P_{D_{2}}\left(\lambda_{m}^{C}\right)+P_{A_{2}}\left(\lambda_{m}^{B}\right)+p_{D_{2}}\left(\lambda_{m}^{C}\right)+p_{A_{2}}\left(\lambda_{m}^{B}\right)\right\}, \\
\tilde{M}_{j K}=M_{j K} \exp \left\{-\Phi_{A_{2}}\left(\lambda_{j}^{C}\right)-\Phi_{D_{2}}\left(\lambda_{K}^{B}\right)-\varphi_{A_{1}}\left(\lambda_{j}^{C}\right)-\varphi_{D_{1}}\left(\lambda_{K}^{B}\right)\right\} .
\end{gathered}
$$

Then we'll construct new fields $\tilde{\Phi}_{\alpha}, \tilde{\varphi}_{\alpha}$ which are equal to

$$
\begin{aligned}
& \tilde{\Phi}_{\alpha}(\lambda)=\Phi_{\alpha}(\lambda)-\left\langle\tilde{0}\left|\Phi_{\alpha}(\lambda)\right| 0\right\rangle, \\
& \tilde{\varphi}_{\alpha}(\lambda)=\varphi_{\alpha}(\lambda)-\left\langle\tilde{0}\left|\varphi_{\alpha}(\lambda)\right| 0\right\rangle .
\end{aligned}
$$

Each of the new fields can be, as before, expressed in terms of "coordinate" and "momentum" by formulae (4.1), in which $\Phi_{\alpha}$ and $\varphi_{\alpha}$ must be replaced by $\tilde{\Phi}_{\alpha}$ and $\tilde{\varphi}_{\alpha}$ correspondingly. Commutation relations $(4.6),(4.7)$ are also valid. The 
only difference is that now all "coordinates" annihilate the dual vacuum $\langle\tilde{0}|$. In terms of $\tilde{\Phi}_{\alpha}$ and $\tilde{\varphi}_{\alpha}$ the matrix $\tilde{M}$ looks as follows:

$$
\begin{aligned}
\tilde{M}_{j K}= & t_{j K}^{C B}+t_{K j}^{B C} \exp \left\{\tilde{\Phi}_{A_{2}}\left(\lambda_{K}^{B}\right)-\tilde{\Phi}_{D_{2}}\left(\lambda_{K}^{B}\right)+\tilde{\Phi}_{D_{2}}\left(\lambda_{j}^{C}\right)-\tilde{\Phi}_{A_{2}}\left(\lambda_{j}^{C}\right)\right\} \times Z\left(\lambda_{K}^{B}\right) Z^{-1}\left(\lambda_{j}^{C}\right) \\
& -\left[t_{j K}^{C B} \exp \left\{\tilde{\Phi}_{A_{1}}\left(\lambda_{j}^{C}\right)+\tilde{\Phi}_{D_{1}}\left(\lambda_{K}^{B}\right)\right\}\right. \\
& \left.+t_{K j}^{B C} \exp \left\{\tilde{\Phi}_{A_{1}}\left(\lambda_{K}^{B}\right)+\tilde{\Phi}_{D_{1}}\left(\lambda_{j}^{C}\right)\right\} \frac{a_{1}\left(\lambda_{K}^{B}\right) d_{1}\left(\lambda_{j}^{C}\right)}{d_{1}\left(\lambda_{K}^{B}\right) a_{1}\left(\lambda_{j}^{C}\right)}\right] \\
& \times Z\left(\lambda_{K}^{B}\right) Z^{-1}\left(\lambda_{j}^{C}\right) \exp \left\{\tilde{\varphi}_{D_{2}}\left(\lambda_{j}^{C}\right)-\tilde{\Phi}_{A_{2}}\left(\lambda_{j}^{C}\right)-\tilde{\varphi}_{A_{1}}\left(\lambda_{j}^{C}\right)\right. \\
& \left.+\tilde{\varphi}_{A_{2}}\left(\lambda_{K}^{B}\right)+\tilde{\varphi}_{D_{3}}\left(\lambda_{K}^{B}\right)-\tilde{\Phi}_{D_{2}}\left(\lambda_{K}^{B}\right)-\tilde{\varphi}_{D_{1}}\left(\lambda_{K}^{B}\right)\right\} \\
& +c \alpha \frac{a_{1}\left(\lambda_{K}^{B}\right)}{d_{1}\left(\lambda_{K}^{b}\right)} Z\left(\lambda_{K}^{B}\right) Z^{-1}\left(\lambda_{j}^{C}\right) \exp \left\{\tilde{\varphi}_{D_{2}}\left(\lambda_{j}^{C}\right)-\tilde{\Phi}_{A_{2}}\left(\lambda_{j}^{C}\right)-\tilde{\varphi}_{A_{1}}\left(\lambda_{j}^{C}\right)\right. \\
& \left.+\tilde{\varphi}_{A_{2}}\left(\lambda_{K}^{B}\right)+\tilde{\varphi}_{D_{3}}\left(\lambda_{K}^{B}\right)-\tilde{\Phi}_{D_{2}}\left(\lambda_{K}^{B}\right)-\tilde{\varphi}_{D_{1}}\left(\lambda_{K}^{B}\right)\right\} .
\end{aligned}
$$

Here

$$
\begin{aligned}
& Z\left(\lambda_{K}^{B}\right)=\frac{a_{2}\left(\lambda_{K}^{B}\right)}{d_{2}\left(\lambda_{K}^{B}\right)} \prod_{m=1}^{N} \frac{h_{K m}^{B B}}{h_{m K}^{C B}}, \\
& Z\left(\lambda_{j}^{C}\right)=\frac{a_{2}\left(\lambda_{j}^{C}\right)}{d_{2}\left(\lambda_{j}^{C}\right)} \prod_{m=1}^{N} \frac{h_{j m}^{C B}}{h_{m j}^{C C}} .
\end{aligned}
$$

The last step is to put $\lambda_{j}^{C}=\lambda_{j}^{B}=\lambda_{j}$ and use (2.13). In this case

$$
Z(\lambda)=\frac{d_{1}(\lambda)}{a_{1}(\lambda)}=e^{i x \lambda}
$$

and we have

$$
\begin{aligned}
&\left\langle 0\left|\prod_{j=1}^{N} C\left(\lambda_{j}\right) \psi^{+}(x) \psi(0) \prod_{j=1}^{N} B\left(\lambda_{j}\right)\right| 0\right\rangle=\left.\prod_{\substack{j, K=1 \\
j \neq K}}^{N} f_{j K} \frac{\partial}{\partial \alpha}\left\langle\tilde{0}\left|\operatorname{det}_{N} V\right| 0\right\rangle\right|_{\alpha=0}, \\
& V_{j K}= c \delta_{j K}\left(L+\sum_{m=1}^{N} K_{j m}\right)+t_{j K}+t_{K j} \exp \left\{i x \lambda_{K j}\right. \\
&\left.+\tilde{\Phi}_{A_{2}}\left(\lambda_{K}\right)-\tilde{\Phi}_{D_{2}}\left(\lambda_{K}\right)-\tilde{\Phi}_{A_{2}}\left(\lambda_{j}\right)-\tilde{\Phi}_{D_{2}}\left(\lambda_{j}\right)\right\} \\
&-\left[t_{j K} \exp \left\{i x \lambda_{K j}+\tilde{\Phi}_{A_{1}}\left(\lambda_{j}\right)+\tilde{\Phi}_{D_{1}}\left(\lambda_{K}\right)\right\}+t_{K j} \exp \left\{\tilde{\Phi}_{A_{1}}\left(\lambda_{K}\right)+\tilde{\Phi}_{D_{1}}\left(\lambda_{j}\right)\right\}\right] \\
& \times \exp \left\{\tilde{\varphi}_{D_{2}}\left(\lambda_{j}\right)-\tilde{\Phi}_{A_{2}}\left(\lambda_{j}\right)-\tilde{\varphi}_{A_{1}}\left(\lambda_{j}\right)+\tilde{\varphi}_{A_{2}}\left(\lambda_{K}\right)\right. \\
&\left.+\tilde{\varphi}_{D_{3}}\left(\lambda_{K}\right)-\tilde{\Phi}_{D_{2}}\left(\lambda_{K}\right)-\tilde{\varphi}_{D_{1}}\left(\lambda_{K}\right)\right\} \\
&+c \alpha \exp \left\{-i x \lambda_{j}+\tilde{\varphi}_{A_{3}}\left(\lambda_{K}\right)+\tilde{\varphi}_{A_{2}}\left(\lambda_{K}\right)-\tilde{\varphi}_{D_{1}}\left(\lambda_{K}\right)\right. \\
&\left.-\tilde{\Phi}_{D_{2}}\left(\lambda_{K}\right)+\tilde{\varphi}_{D_{2}}\left(\lambda_{j}\right)-\tilde{\Phi}_{A_{2}}\left(\lambda_{j}\right)\right\}
\end{aligned}
$$

where $\lambda_{K j}=\lambda_{K}-\lambda_{j}$ and

$$
K_{j m}=K\left(\lambda_{j}, \lambda_{m}\right)=\frac{2 c}{\left(\lambda_{j}-\lambda_{m}\right)^{2}+c^{2}} .
$$


Formula (5.8) gives us an expression for the correlator of fields in the $N$-particle state. $|\Phi\rangle$

Consider now the correlator of fields in the ground state of the Hamiltonian:

$$
\left\langle\psi^{+}(x) \psi(0)\right\rangle=\frac{\left\langle\Phi\left|\psi^{+}(x) \psi(0)\right| \Phi\right\rangle}{\langle\Phi \mid \Phi\rangle}
$$

This correlator can be obtained from (5.7) in the thermodynamic limit: $N \rightarrow \infty, L \rightarrow \infty, N / L=$ const. The eigenstate $|\Omega\rangle$ is a Dirac sea. Momenta of particals $\lambda_{j}$ are bounded by the Fermi momentum $q:\left|\lambda_{j}\right|<q$. They are described by the distribution density $\varrho(\lambda)$ which satisfies the equation

$$
\left(1-\frac{1}{2 \pi} \hat{K}\right) \varrho(\lambda)=\frac{1}{2 \pi}
$$

Here $\hat{K}$ is the integral operator, acting in the interval $[-q, q]$ with the kernel $K(\lambda, \mu)$ [see (5.9)]. The square of the norm of the eigenfunction $|\Phi\rangle$ was calculated in [7]:

$$
\langle\Phi \mid \Phi\rangle=\prod_{j=1}^{N}\left(2 \pi c L \varrho\left(\lambda_{j}\right)\right) \prod_{\substack{j, K=1 \\ j \neq K}}^{N} f_{j K} \operatorname{det}\left(1-\frac{1}{2 \pi} \hat{K}\right)
$$

To write down the expression for the correlator in thermodynamic limit it is sufficient to replace the sum $\sum_{m=1}^{N} K_{j m}$ in (5.8) by the correspondent integral. Using
(5.11) one have

$$
L+\sum_{m=1}^{N} K_{j m} \rightarrow L\left(1+\int_{-q}^{q} K\left(\lambda_{j}, \mu\right) \varrho(\mu) d \mu\right)=2 \pi L \varrho\left(\lambda_{j}\right)
$$

so we obtain

$$
\left\langle\psi^{+}(x) \psi(0)\right\rangle=\left.\frac{\partial}{\partial \alpha} \frac{\left\langle\tilde{0}\left|\operatorname{det}\left(1+\frac{1}{2 \pi c} \hat{V}_{0}\right)\right| 0\right\rangle}{\operatorname{det}\left(1-\frac{1}{2 \pi} \hat{K}\right)}\right|_{\alpha=0}
$$

where $\hat{V}_{0}$ is the integral operator, acting in the interval $[-q, q]$ with the kernel:

$$
\begin{aligned}
V_{0}(\lambda, \mu)= & t(\lambda, \mu) e^{\frac{i x}{2}(\lambda-\mu)}+t(\mu, \lambda) \\
& \times \exp \left\{\frac{i x(\mu-\lambda)}{2}+\tilde{\Phi}_{A_{2}}(\lambda)-\tilde{\Phi}_{D_{2}}(\lambda)+\tilde{\Phi}_{D_{2}}(\mu)-\tilde{\Phi}_{A_{2}}(\mu)\right\} \\
& -\left[t(\lambda, \mu) \exp \left\{\frac{i x(\mu-\lambda)}{2}+\tilde{\Phi}_{A_{1}}(\lambda)+\tilde{\Phi}_{D_{1}}(\mu)\right\}\right. \\
& \left.+t(\mu, \lambda) \exp \left\{\frac{i x(\lambda-\mu)}{2}+\tilde{\Phi}_{A_{1}}(\mu)+\tilde{\Phi}_{D_{1}}(\lambda)\right\}\right]
\end{aligned}
$$




$$
\begin{aligned}
& \times \exp \left\{\tilde{\varphi}_{D_{2}}(\lambda)-\tilde{\varphi}_{A_{1}}(\lambda)-\tilde{\Phi}_{A_{2}}(\lambda)+\tilde{\varphi}_{A_{2}}(\mu)\right. \\
& \left.+\tilde{\varphi}_{D_{3}}(\mu)-\tilde{\varphi}_{D_{1}}(\mu)-\tilde{\Phi}_{D_{2}}(\mu)\right\} \\
& +\alpha c \exp \left\{-\frac{i x}{2}(\lambda+\mu)+\tilde{\varphi}_{D_{2}}(\lambda)-\tilde{\Phi}_{A_{2}}(\lambda)+\tilde{\varphi}_{A_{3}}(\lambda)\right. \\
& \left.+\tilde{\varphi}_{A_{2}}(\mu)-\tilde{\varphi}_{D_{1}}(\mu)-\tilde{\Phi}_{D_{2}}(\mu)\right\}
\end{aligned}
$$
[8]

In the case of finite temperature the correlation function of fields is equal to

$$
\left\langle\psi^{+}(x) \psi(0)\right\rangle_{T}=\frac{\left\langle\Phi_{T}\left|\psi^{+}(x) \psi(0)\right| \Phi_{T}\right\rangle}{\left\langle\Phi_{T} \mid \Phi_{T}\right\rangle},
$$

where $\left|\Phi_{T}\right\rangle$ is one of the eigenfunctions, describing the state of thermodynamic equilibrium. The distribution density is equal to

$$
\begin{aligned}
2 \pi \varrho(\lambda) \theta^{-1}(\lambda) & =1+\int_{-\infty}^{\infty} K(\lambda, \mu) \varrho(\mu) d \mu \\
\theta^{-1}(\lambda) & =1+\exp \left[\frac{\varepsilon(\lambda)}{T}\right] .
\end{aligned}
$$

$T$ is the temperature, and $\varepsilon(\lambda)$ - density of energy:

$$
\varepsilon(\lambda)=\lambda^{2}-h+\frac{T}{2 \pi} \int_{-\infty}^{\infty} K(\lambda, \mu) \ln \left(1+\exp \left[-\frac{\varepsilon(\mu)}{T}\right]\right) d \mu .
$$

It is easy to see that in the case of finite temperature the correlator is equal to

$$
\left\langle\psi^{+}(x) \psi(0)\right\rangle_{T}=\left.\frac{\partial}{\partial \alpha} \frac{\left\langle\tilde{0}\left|\operatorname{det}\left(1+\frac{1}{2 \pi c} \hat{V}_{T}\right)\right| 0\right\rangle}{\operatorname{det}\left(1-\frac{1}{2 \pi} \hat{K}_{T}\right)}\right|_{\alpha=0}
$$

Here

$$
\begin{aligned}
& V_{T}(\lambda, \mu)=V_{0}(\lambda, \mu) \sqrt{\theta(\lambda) \theta(\mu)}, \\
& V_{T}(\lambda, \mu)=K(\lambda, \mu) \sqrt{\theta(\lambda) \theta(\mu)} .
\end{aligned}
$$

Note that in the point of free fermions $(c=\infty)$ all dual fields can be put equal to zero, because all "coordinates" and "momenta" commute. The kernel $V_{0}$ symplifies:

$$
\left.c^{-1} V_{0}\right|_{c=\infty}=-\frac{2}{\pi} \frac{\sin \frac{x}{2}(\lambda-\mu)}{\lambda-\mu}+\frac{\alpha}{2 \pi} e^{-\frac{i x}{2}(\lambda+\mu)} .
$$

In such a form this answer was obtained before in [1].

In conclusion let us notice that the method of dual fields, described in this paper and before in $[5,6]$, can be easily generalized for calculation of multipoint correlation functions. It also gives us the possibility to calculate correlators in models with an $R$-matrix of $X X Z$ type. 


\section{References}

1. Lenard, A.: One-dimensional impenetrable bosons in thermal equilibrium. J. Math. Phys. 7, 1268-1272 (1966)

2. Jimbo, M., Miwa, T., Mori, Y., Sato, M.: Density matrix of an impenetrable Bose gas and the fifth Painleve transcendent. Physica D 1, 80-158 (1980)

3. Its, A.R., Izergin, A.G., Korepin, V.E.: Long-distance asymptotics of temperature correlators of Impenetrable bose gas. Commun. Math. Phys. 130, 471-488 (1990)

Its, A.R., Izergin, A.G., Korepin, V.E., Slavnov, N.A.: Differential equations for quantum correlation functions. Int. J. Mod. Phys. B 4, 1003-1037 (1990)

4. Izergin, A.G., Korepin, V.E.: The quantum inverse scattering method approach to correlation functions. Commun. Math. Phys. 113, 117-190 (1987)

5. Korepin, V.E.: Generating functional of correlation functions for nonlinear Schrödinger equation. Funct. Anal. 23, (1), 15-23 (1989)

6. Korepin, V.E.: Calculations of norms of Bethe wave functions. Commun. Math. Phys. 86, 391-418 (1982)

7. Bogoliubov, N.M., Korepin, V.E.: Correlation functions of one-dimensional Bose gas in thermal equilibrium. Theor. Math. Phys. 60, (2), 262-269 (1984)

8. Korepin, V.E., Slavnov, N.A.: The time dependent correlation function of an impenetrable Bose gas as a Fredholm minor. Commun. Math. Phys. 129, 103 (1990)

Communicated by N.Yu. Reshetikhin 